\title{
EL MANEJO DE DESECHOS SÓLIDOS EN LAS INSTITUCIONES EDUCATIVAS
}

\author{
AUTORES: Graciela Celeste Pincay Franco ${ }^{1}$ \\ Juber Roderich Bravo Muñoz ${ }^{2}$ \\ Anaysa Alvarez Luna ${ }^{3}$ \\ Flor Isdalba Guerra Castro ${ }^{4}$ \\ Adis Anicia Luna Baez ${ }^{5}$
}

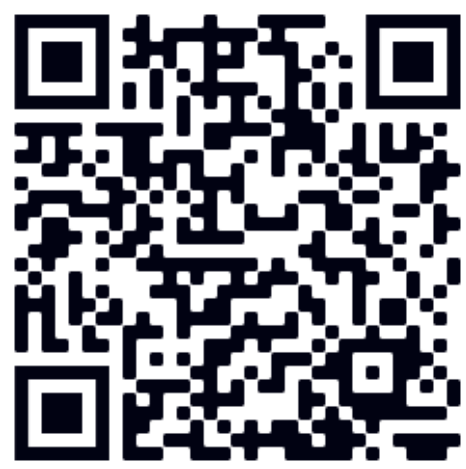

DIRECCIÓN PARA CORRESPONDENCIA: graciela.pincay@educacion.gob.ec

Fecha de recepción: 12/06/2019

Fecha de aceptación: 30/11/2019

\section{RESUMEN}

El manejo y disposición de residuos sólidos en el mundo constituye un gran problema y es grave en las ciudades de los países en desarrollo y de este fenómeno social no se excluyen las instituciones educativas, es indispensable que los estudiantes se actualicen sobre este tema tan actual. Esta investigación tiene como objetivo evaluar las actividades relacionadas con el manejo de desechos sólidos de los estudiantes para mejorar el cuidado del medio ambiente en las instituciones docentes, es un estudio cualitativo, que utiliza la metodología exploratoria, de campo y bibliográfica, a través de métodos teóricos como: el análisis-síntesis, la abstracción, el histórico lógico para estudiar cómo evolucionó este proceso, además los empíricos como: entrevistas, encuestas, observaciones, revisión de documentos a directivos, docentes, estudiantes y padres, para constatar las insuficiencias que se presenta con respecto a la sistematización del manejo de desechos sólidos en la institución. Se diseñó una propuesta de actividades para mejorar el manejo de desechos sólidos y el cuidado del medio ambiente, orientada a fomentar la comprensión de la problemática y sus consecuencias en la salud de los estudiantes y su impacto negativo en el medio ambiente.

PALABRAS CLAVE: actividades; ambiente; cuidado

\section{SOLID WASTE MANAGEMENT IN EDUCATIONAL INSTITUTIONS}

\section{ABSTRACT}

\footnotetext{
${ }^{1}$ Lic. Ciencias de la Educación Mención Ecología y Medioambiente. Mg Orientación Educativa. Docente de U. E. Judith Iza de Antón. Jipijapa. Manabí. Ecuador. graciela.pincay@educacion.gob.ec

2 Lic. Ciencias de la Educación. Cultura Física. Mg. Docencia Universitaria e Investigación Educativa. Mg. Orientación Educativa. Docente U. E. Quince de Octubre. Jipijapa. Manabí. Ecuador juber.bravo@educacion.gob.ec ${ }^{3}$ Doctora en Medicina. Especialidad en Ginecobstetricia. Jipijapa. Universidad de Ciencias Médicas. Granma. Cuba aaluna1992@gmail.com

${ }^{4}$ Ingeniero Agropecuario Docente de U. E. Judith Iza de Antón. Jipijapa. Manabí. Ecuador flor.guerra@educacion.gob.ec

${ }^{5}$ Licenciada en Química. Magister en Ciencias de la Educación y Dirección Educacional. Docente Universidad Estatal del Sur de Manabí. adis.luna@unesum.edu.ec orcid-id 0000-0001-6260-3454
} 
Graciela Celeste Pincay Franco, Juber Roderich Bravo Muñoz, Anaysa Alvarez Luna...

The management and disposal of solid waste in the world is a major problem and is serious in the cities of developing countries and this social phenomenon does not exclude educational institutions, it is essential that students are updated on this subject so current. This research aims to evaluate students' solid waste management activities to improve environmental care in educational institutions. It is a qualitative study that uses exploratory methodology, field and bibliographic, through theoretical methods such as: analysis-synthesis, abstraction, logical history to study how this process evolved, in addition to empirical ones such as: interviews, surveys, observations, review of documents to managers, teachers, students and parents, to see what are the shortcomings that arise with respect to the systematization of solid waste management in the institution. A proposal for activities was designed to improve solid waste management and environmental care, aimed at promoting understanding of the issues and their impact on student health and its negative impact on the environment.

KEYWORDS: activities; ambient; watch out

\section{INTRODUCCIÓN}

El manejo de los desechos sólidos se ha convertido en un problema a nivel mundial debido a que cada vez se generan más residuos y la educación ambiental es escasa. Es conocido que la descomposición de la basura conlleva a la reproducción de vectores transmisores de enfermedades aparte de generar malos olores y deteriorar el paisaje y por ende el ambiente. Es así como la importancia del manejo de desechos sólidos se convierte en un tema diario y de práctica educativa permanente, en muchos casos bajo proyectos genera ganancias y se ayuda al ambiente en cualquiera de sus ecosistemas.

En Ecuador se han puesto en práctica proyectos para el desarrollo sostenible basados en la transformación e innovación de los desechos reutilizables direccionado a los estudiantes universitarios y aprovechando su conocimiento fresco e innovador para contribuir de esta manera a la mejora del ambiente. La necesidad de resolver el problema desconocimiento del manejo de desechos sólidos es importante para mantener un entorno limpio y sin contaminación iniciando en las instituciones educativas para fortalecer los valores de responsabilidad y el cuidado del medio ambiente para que se desarrolle desde temprana edad (Arichabala, 2014).

Un ambiente limpio aprovechando de las bondades de la naturaleza en todas las instituciones docentes influye en que la calidad de vida sea mejor porque se convertirá en una buena costumbre el correcto manejo de los desechos sólidos y se promoverá al correcto reciclaje de los desechos orgánicos e inorgánicos; sin olvidar que gracias a este aprendizaje se podrán evitar desequilibrios en la salud de los estudiantes que seguramente afectarán su desarrollo físico e intelectual.

La aplicación de estas actividades constituye un trabajo que posibilitará cambios en la salud de los estudiantes, su entorno natural y la comunidad, que se logrará con la práctica diaria, creativa y eficaz de todos; para iniciar la aplicación de estas actividades educativas con los estudiantes, se realizaron talleres de motivación para involucrar a todos los docentes, estudiantes, padres de familias y comunidad, se realizan además charlas y campañas de limpieza, porque los estudiantes necesitan de este nivel de ayuda para el desarrollo y la sistematización de habilidades que propicien el conocimiento de la clasificación de los desechos sólidos y cómo estos por su acumulación o mal manejo afectan la salud del ser vivo. Dentro de las actividades juegan un papel destacado: las

2 UNESUM-Ciencias. Publicación cuatrimestral. Vol. 3, Año 2019, No. 3 (Septiembre - Diciembre) 
charlas, campañas y excursiones, acciones que deben ser permanentes para evitar la contaminación por basura y que esta basura contamine el aire y el agua de la escuela.

La solución de los problemas ambientales para la educación de las nuevas generaciones en las instituciones educativas y de la población nunca será $100 \%$ correcta a problemas de la comunidad, las acciones y actividades que se planifican como, la solución adecuada, que depende de los propios valores y prioridades de los estudiantes, docentes y participantes de la comunidad (Plan Nacional de Desarrollo, 2017-2021).

Es importante la enseñanza sobre manejo de residuos sólidos en las instituciones educativas, que significa cambiar, fortalecer la esencia de sus conocimientos sobre este tema para que los estudiantes aprendan y desarrollen habilidades en la clasificación de desechos, guiados por sus docentes, y lo que aprenden sobre el problema de los residuos, compararlo con la importancia de conocer los problemas humanos relacionados con el uso racional de los recursos naturales y el cuidado del medio ambiente (Martínez \& Balaguer, 2016).

La evaluación de la secuencia enseñanza - aprendizaje de este tema tan vital, no puede estar sólo basada en datos que describen el posterior grado de participación de los estudiantes y docentes en el reciclaje, la evaluación debe abordar el valor educativo, esta debe dirigirse, entonces, hacia el grado del nivel de lo aprendido para comprender la base del problema del manejo de residuos, en qué medida es capaz de utilizar este conocimiento para comprender otros problemas ambientales (Martínez \& Balaguer, 2016).

A lo largo de la vida de la educación ambiental han tenido lugar frecuentes discusiones sobre si la educación ambiental debería enseñarse como una asignatura separada o integrarse en las materias existentes. Incluso aunque este debate no está establecido en todos los países, en conjunto, la mayoría de los educadores ambientales defienden el proceso interdisciplinario para especificar aspectos relativos a la educación ambiental en la mayoría de los programas de las distintas asignaturas (desde 1994). Aceptar tener la educación ambiental integrada en las asignaturas existentes significa que es una necesidad urgente tener una imagen clara de las particularidades de la educación ambiental (Follari, 2001).

Sobre las ventajas que ofrece el desarrollo de la interdisciplinariedad, que también son asumidos de J. Fiallo, se analizó la importancia de este proceso ya que elimina las fronteras entre las disciplinas, posibilita el aumento de la motivación en los estudiantes, así como una interrelación entre los conceptos que se desarrollan, y las definiciones con que opera el estudiante, permitiendo, además la sistematización de las habilidades y educando un pensamiento lógico, reflexivo e integrador a la vez que despierta el interés de los docentes y propicia entre ellos mejores relaciones de trabajo ( Explora Natura , 2019).

Estos fundamentos teóricos se aplicaron en las unidades de aprendizaje y se las relacionó con la problemática ambiental. Se diseñó las actividades relacionadas con el manejo de desechos sólidos para mejorar la educación ambiental y las actividades inherentes a las competencias genéricas y disciplinares, en las que se consideraron los parámetros que se muestran en la figura 1 (Cadmea \& et al, 2015). 
Graciela Celeste Pincay Franco, Juber Roderich Bravo Muñoz, Anaysa Alvarez Luna...

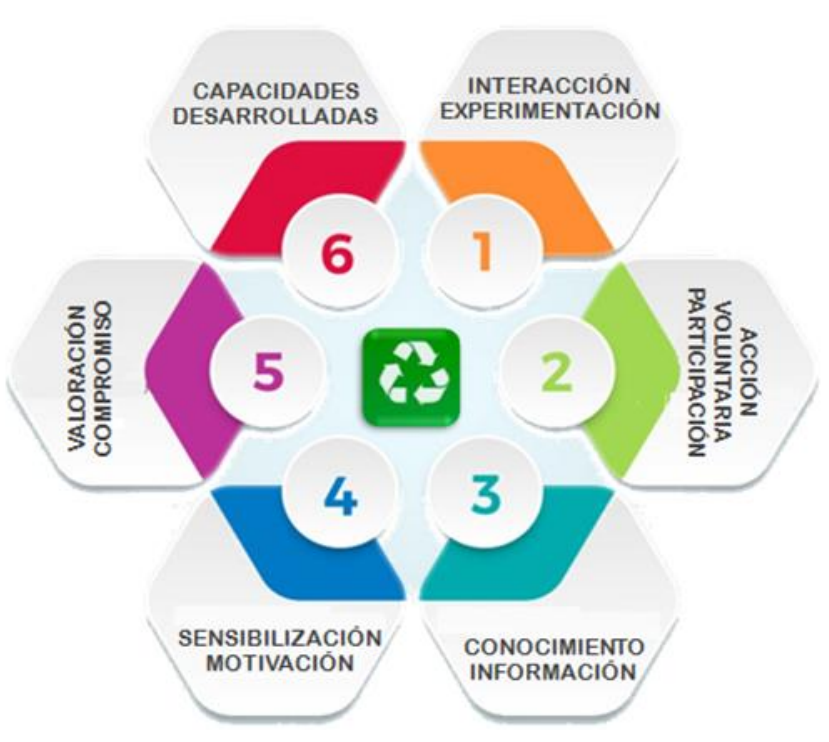

Figura 1. Parámetros relacionados con la problemática ambiental.

\section{DESARROLLO}

Dentro de la Estrategia Nacional de Educación Ambiental uno delos objetivos esenciales es fomentar la educación ambiental como parte de la formación integral del estudiantado de los niveles de Educación Inicial, General Básica y Bachillerato para impulsar metodologías, técnicas y recursos de educación y comunicación ambiental, que faciliten el desarrollo de conocimientos, comportamientos y prácticas socio-ambientales que estimulen una relación positiva y coherente entre el ser humano, integrando los saberes y ciencias de los diversos pueblos y nacionalidades del Ecuador (Ministerio del Ambiente, 2019)

El proceso de investigación cumplió con esta estrategia nacional, está relacionado con la evaluación de las actividades relacionadas con el manejo de desechos sólidos de los estudiantes para mejorar el cuidado del medio ambiente en las instituciones docentes es un estudio cualitativo, la metodología utilizada es descriptiva, y acción-participativa.

En este proceso investigativo sobre una realidad social, se hace una descripción del objeto de estudio y de sus factores implícitos, para realizar un análisis de esa cualificación realizada, para así tener un acercamiento a la realidad de esta problemática ambiental; que está sujeto a las acciones por parte de los docentes ejecutores esta investigación, en cuanto a la realización de las observaciones y encuentros con los estudiantes y la comunidad educativa que hacen parte del proceso evolutivo del proyecto, participando en su desarrollo.

Para el desarrollo de este trabajo de investigación se pudo obtener la información necesaria para describir el problema del manejo de los desechos sólidos y su relación con el cuidado del medio ambiente; en la decisión sobre el mejor modo de obtener los datos y de quién o quiénes obtenerlos. Se toman en el campo, ya que los participantes del estudio nos resultan desconocidos cuando se inicia este proceso y es la propia información obtenida la que va guiando el muestreo y el diagnóstico de la realidad en el contexto estudiado (Sampieri, 2018). 
En una primera etapa se realizó la búsqueda de información secundaria, por medio de una revisión bibliográfica y documental, a efectos de revisar los principales referentes conceptuales relacionados con la temática de investigación.

La metodología empleada en la investigación corresponde al tipo exploratorio, que ayuda a familiarizar a los estudiantes con el contexto de la problemática, en este caso el sector de la ciudad de Jipijapa y a partir de ello recopilar información específica sobre las principales características del entorno (Sampieri, 2018).

Cabe destacar que la recopilación de información primaria, que complementa la información de las fuentes secundarias se realizó por medio de la observación no participante y la aplicación de dos técnicas de investigación con fin de recolectar información más concreta: una encuesta dirigida a los moradores de la comunidad, y una entrevista en la que se formuló a los dirigentes una serie de preguntas referentes al tema estudiado (Sampieri, 2018).

En el proceso de la investigación se realizó un diagnóstico situacional del sector, utilizando fichas de observación, encuestas, entrevistas para establecer la realidad educativa ambiental y el manejo de los residuos sólidos manejo de los residuos sólidos. Este sistema de actividades educativas permite disminuir la contaminación de la naturaleza y mejorar la imagen de las instituciones educativas y la comunidad.

En esta investigación se comprobó que en el centro si existía un programa que norma o estableciera las actividades a realizar en el plano medio ambiental, el director de dicho centro respondió, que no existe, o sea, una planificación para ejecutar actividades.

Se aplico una encuesta a los estudiantes de la escuela Judith Iza de Antón, donde se realizó el estudio de campo, se aplicó encuesta de preguntas abiertas y cerradas que proporcionaron la información necesaria, para diagnosticar las necesidades de planificar, ejecutar y evaluar actividades relacionadas con el manejo de desechos sólidos y su relación con la educación ambiental en la institución educativa.

La primera parte (diagnóstico inicial) que trata sobre el conocimiento del manejo medio ambiente, como lo definen y que problemas de su municipio conocen. En la segunda parte, se indagó si realizan actividades de educación ambiental en las clases de Educación Física y actividades físicas extradocentes y extraescolares o la disposición a realizar actividades de este tipo.

La población está formada 230 estudiantes y 20 docentes, de la institución educativa su función básica es determinar el estudio de las variables en dicha institución. El muestreo realizado es probabilístico (aleatorio): fueron muestreados 70 estudiantes de nivel básico superior y sus 10 docentes de la sesión vespertina, en este muestreo todos los integrantes de la población pueden formar parte de la muestra, tienen probabilidad positiva de formar parte de la muestra, por lo tanto, es el tipo de muestreo a utilizar en el proceso de investigación, por ser el riguroso y científico (Sampieri, 2018).

Existió una relación directa entre los parámetros estudiados en el proceso de evaluación sobre: sobre lo conocimiento-información y sensibilización-motivación. Se observó trabajo en equipo disciplinario, metódico, coherente y responsable en el proceso de búsqueda y selección de la información que permitieron comprender de manera clara y precisa la problemática estudiada y sensibilizar su participación humana en disminuir el deterioro de los recursos naturales y a generar motivación para encontrar soluciones a la misma. 
Graciela Celeste Pincay Franco, Juber Roderich Bravo Muñoz, Anaysa Alvarez Luna...

Se desarrolló las capacidades de experimentación e interacción, que determinó que la actividad ejecutada facilitó la exteriorización de los pensamientos, criterios, necesidades, sugerencias e ideas que les permitieron integrarse en el trabajo; asimilar de manera personal la información obtenida para planificar, diseñar y experimentar diferentes actividades; seguir las directrices del docente guía y del líder del grupo; y, fortalecer la capacidad para generar soluciones viables ante la problemática ambiental analizada, sobre la base del paradigma constructivista del aprendizaje, donde los estudiantes guiados por el docente es capaz de hacer y de forma creativa aplicar estas actividades en la práctica para mejorar el manejo de los desechos sólidos y con ello el cuidado del medio ambiente (Carranza, 2005).

Se logró la valoración-compromiso y acción voluntaria-participación que se manifestó en el fortalecimiento de la relación docente - estudiante, se mantuvo un compromiso permanente para alcanzar la meta propuesta fundamentada en la participación voluntaria.

Se valoró la expresión práctica de la capacidad humana para corregir errores y la necesidad de generar cultura, responsabilidad y ética ambiental a nivel individual, comunitario en el cuidado del medio ambiente en los estudiantes.

Se realizó una jornada de concientización ambiental sobre el manejo adecuado de los residuos sólidos y la aplicación de una encuestas a la muestra seleccionada donde se evidencia el nivel de conocimiento que tienen sobre el manejo de los residuos sólidos, constatando el índice cognitivo sobre el impacto ambiental que genera el mal uso de los residuos sólidos.

Se fortaleció el rol docente en el acto de educar y concienciar a las diferentes generaciones para desarrollar competencias éticas y reorientar las prácticas de los grupos, al reconocer en la interacción maestro/estudiante un ejercicio de construcción de significados compartidos acerca de la educación ambiental orientada hacia una conciencia ambiental, además de su incidencia en las formas de valorar y actuar frente al ambiente, en una reinvención de relaciones más armónicas entre las comunidades y de estas con el entorno.

\section{CONCLUSIONES}

La observación a la institución y a su entorno determinó el impacto visual negativo que produce el mal uso de los residuos sólidos en la institución, arrojando así un diagnóstico de gran utilidad a la presente investigación pues permiten planificar, ejecutar y evaluar las actividades en la institución educativa.

Se identificó el interés y compromiso entre el docente-estudiante durante el proceso de planificación y ejecución de las actividades educativas para mejorar la manipulación de los desechos sólidos para proteger el medio ambiente de las instituciones y la comunidad.

Los estudiantes condujeron adecuadamente la selección y recopilación bibliográfica acorde a la necesidad, se demostró respeto y cumplimiento a las disposiciones, se promovió la investigación (causa-efecto) de la problemática y el análisis metódico a nivel cualitativo y cuantitativo para definir soluciones.

Se demostró capacidad de liderazgo, trabajo en equipo y predisposición en la socialización de la información durante la realización de los resultados de las actividades realizadas para promover cultura, ética y responsabilidad ambiental en los docentes y estudiante a nivel institucional y de las unidades educativas participantes. 
La educación ambiental puede contribuir al manejo de los desechos sólidos y puede generar nuevos comportamientos, actitudes, valores y creencias que impulsen el desarrollo social, productivo y creador; como consecuencia puede ser el medio para el logro de nuevas relaciones entre los seres humanos y esencialmente en la formación de las nuevas generaciones, responsables del cuidado de este proceso en el futuro.

\section{REFERÉNCIAS BIBLIOGRÁFICAS}

Explora Natura . (24 de mayo de 2019). Interdisciplinariedad Educación Ambiental. Obtenido de https://www.exploranatura.com/interdisciplinariedad-educacion-ambiental-2/

Arichabala. (2014). Los 10 Mejores Proyectos sobre el cuidado del medio ambiente y el manejo de desechos sólidos. Quito: ODEBRECHT.

Cadmea, M., \& et al. (2015). La Educación Ambiental en la Educación Superior. Quito: Facultad de Ingenieria Ambiental y recursos naturales.

Carranza, R. (2005). El constructivismo como estrategia educativa:formación profesional . Ensayo, 5-12.

Follari, R. (2001). Estudios culturales, transdisciplinariedad e interdisciplinariedad. Unidad y Praxis.

Martínez , \& Balaguer. (2016). Universidad saludable: una estrategia de promoción de la salud y salud en todas las políticas para crear un entorno de trabajo saludable. Barcelona: Arch Prev Riesgos Labor vol.19 no.3.

Ministerio del Ambiente. (junio de 24 de 2019). Dirección de Información, Seguimiento y Evaluación -DISE. Obtenido de maeduca@ambiente.gob.ec

Plan Nacional de Desarrollo. (2017-2021). Sobre los aprendizajes compartidos: Presencia e iniciativas de las instituciones a favor de la educación ambiental. Quito: Educación para el desarrollo sostenible.

Sampieri. (2018). Metodología de la investigación: las rutas cuantitativa, cualitativa y mixta. Mexico: McGraw Hill Mexico 
Graciela Celeste Pincay Franco, Juber Roderich Bravo Muñoz, Anaysa Alvarez Luna...

8 UNESUM-Ciencias. Publicación cuatrimestral. Vol. 3, Año 2019, No. 2 (Mayo - Agosto) 